\title{
Safety and complications of medical thoracoscopy in the management of pleural diseases
}

Yun-Yan Wan ${ }^{\dagger}$, Cong-Cong Zhai ${ }^{\dagger}$, Xin-Shan Lin®, Zhou-Hong Yao, Qing-Hua Liu, Ling Zhu, De-Zhi Li, Xi-Li Li, Ning Wang and Dian-Jie Lin ${ }^{*}$

\begin{abstract}
Background: Medical thoracoscopy is considered an overall safe procedure, whereas numbers of studies focus on complications of diagnostic thoracoscopy and talc poudrage pleurodesis. We conduct this study to evaluate the safety of medical thoracoscopy in the management of pleural diseases and to compare complications in different therapeutic thoracoscopic procedures.

Methods: A retrospective study was performed in 1926 patients, 662 of whom underwent medical thoracoscopy for diagnosis and 1264 of whom for therapeutic interventions of pleural diseases. Data on complications were obtained from the patients, notes on computer system, laboratory and radiographic findings. Chi-square test was performed to compare categorical variables and Fisher's exact test was used for small samples.

Results: The mean age was $51 \pm 8.4$ (range 21-86) years and 1117 (58\%) were males. Diagnostic procedure was taken in 662 (34.4\%) patients, whereas therapeutic procedure was taken in 1264 (65.6\%) patients. Malignant histology was reported in 860 (44.6\%) and 986 (51.2\%) revealed benign pleural diseases. Eighty patients (4.2\%) were not definitely diagnosed and they were considered as unidentified pleural effusion. One patient died during the creation of artificial pneumothorax, and the causes of death were supposed as air embolism or an inhibition of phrenic motoneurons and circulatory system. Complication of lung laceration was found in six patients (0.3\%) and reexpansion pulmonary edema was observed in two patients (0.1\%). Higher incidence of prolonged air leak was observed in bulla electrocoagulation group, in comparison with pleurodesis group. Moreover, pain and fever were the most frequently complications in pleurodesis group and cutaneous infection in entry site was the most frequently reported complication in pleural decortication of empyema group.

Conclusions: Medical thoracoscopy is generally a safe and effective method, not only in the diagnosis of undiagnosed pleural effusions, but also in the management of pleural diseases. Mastering medical thoracoscopy well, improving patient management after the procedure and attempts to reduce the occurrence of post-procedural complications are the targets that physicians are supposed to achieve in the future.
\end{abstract}

Keywords: Medical thoracoscopy, Pleural diseases, Safety, Complication

\footnotetext{
*Correspondence: lindianjie@126.com

†Yun-Yan Wan and Cong-Cong Zhai contributed equally to this work and

should be considered co-first authors

Department of Respiratory Medicine, Shandong Provincial Hospital affiliated

to Shandong University, Shandong University, No. 324 Jingwuweiqi Road,

Jinan, Shandong Province 250021, People's Republic of China
}

(c) The Author(s). 2019 Open Access This article is distributed under the terms of the Creative Commons Attribution 4.0 International License (http://creativecommons.org/licenses/by/4.0/), which permits unrestricted use, distribution, and reproduction in any medium, provided you give appropriate credit to the original author(s) and the source, provide a link to the Creative Commons license, and indicate if changes were made. The Creative Commons Public Domain Dedication waiver (http://creativecommons.org/publicdomain/zero/1.0/) applies to the data made available in this article, unless otherwise stated. 


\section{Background}

Thoracoscopy was first used in 1865 by Francis Richard Cruise, a physician from Ireland [1] and in 1866 Dr. Samuel Gordon reported its use to examine the pleural space in a girl with empyema [2]. In 1998, for the first time, semirigid thoracoscope was evaluated as a new instrument for the examination of thoracic cavity [3]. Compared with surgical thoracoscopy, medical thoracoscopy (MT) requires local anaesthesia under conscious sedation and usually one or two points of entry with a result of less invasive and less expensive [4].

Medical thoracoscopy is initially used for diagnosing exudative pleural effusion [5], and it has been proved to be superior to fluid cytology and 'blind' closed needle pleural biopsy [6-8] with an accuracy of $98-100 \%$ [9]. There are several therapeutic applications of MT, including pleurodesis, treatment of infected pleural space, forceps lung biopsy and sympathectomy $[10,11]$.

When performed by experienced physicians under local anesthesia and moderate sedation, medical thoracoscopy is considered an overall safe procedure [12-14]. Complications of MT can be divided into major and minor complications. According to a comprehensive study, major complications (pneumonia, hemorrhage, empyema, bronchopleural fistula, port site tumor growth, postoperative pneumothorax or air leak) occurred in 86/4736 cases (1.8\%). Minor complications (fever, minor haemorrhage, subcutaneous emphysema, operative skin site infection, atrial fibrillation, hypotension during procedure) occurred in 177/2411 procedures (7.3\%) [14]. However, few clinical trials ever reported complications of MT in the management of pleural diseases.

The aim of present research is to evaluate the safety of $\mathrm{MT}$ and to compare incidence of complications in different therapeutic thoracoscopic procedures.

\section{Methods}

\section{Patients}

A retrospective study was performed in 1926 patients who underwent MT for diagnostic and therapeutic purposes in the department of Respiratory Medicine in Shandong Provincial Hospital affiliated to Shandong University from 1992 to 2017.

Pre-thoracoscopic investigations were conducted as the following items: pleural fluid analysis, chest X-ray and CT, chest ultrasonography (US), ECG, and routine laboratory investigations. Exclusion criteria: severe COPD and respiratory insufficiency, inability to tolerate partial or complete unilateral collapse of the lung, severe contralateral lung or pleural involvement, uncontrollable cough, a fused pleural space with extensive adhesions.

Patients' data were obtained from the hospital's Patient Administration System Database. The following clinical variables were recorded: data on demographics, physical, laboratory and radiologic findings, instruments (semi- rigid or rigid medical thoracoscopy), thoracoscopic findings and therapeutic procedures, histopathologic results, microbiological results, complications of MT, and treatment evaluation of patients.

\section{Medical Thoracoscopy}

Medical thoracoscopy was conducted with a rigid thoracoscope (OLYMPUS; GERMANY) or a semirigid thoracoscope (OLYMPUS MEDICAL SYSTEMS CORP., Tokyo, Japan). Patients were placed in the lateral decubitus position with conscious sedation using diazepam or benzodiazepine. The lateral area of chest was sterilized and draped, $10 \mathrm{~mL}$ of $2 \%$ lidocaine (Silver Lake Shiyao Pharmaceutical Co.Ltd.; Yuncheng, Shanxi Province, China) with $0.5 \mathrm{mg}$ of epinephrine [GrandPharma (China) Co., Ltd.; Wuhan, Hubei Province] was administered to the selected intercostal space for local anesthesia. Oxygen via nasal cannula was administered with 3-4 L/min to maintain $\mathrm{SpO} 2>90 \%$. During the entire procedure, blood pressure, cardiac rhythm, and oxygen saturation were monitored by continuous electrocardiograph and percutaneous oximetry monitoring. Thoracoscopy was performed with single or double entry ports $[15,16]$. The single port, in the midaxillary line between the 4th and 7th intercostal spaces, was commonly used for diagnostic procedure and pleural biopsy, while double ports were used for pleural decortication of empyema, to facilitate adhesiolysis, to control bleeding, to perform pleurodesis or bulla electrocoagulation. The second port was chosen depending on intrathoracic condition, usually in line with the first entry and located one or two intercostal spaces superior or inferior to the first.

Biopsies were taken from parietal pleura for histopathology and microbiological examinations. When MT was used for pleurodesis, 3-5 g of talc was insufflated into the pleural cavity with a catheter and bulb syringe. Bulla electrocoagulation was commonly implemented in patients with recurrent pneumothorax [17]. When extensive adhesions were found under thoracoscopy and precluded lung re-expansion, adhesiolysis with biopsy forceps or electrothermal forceps was performed. In addition, thoracoscopy was done early in the course of empyema where fluid could be easily evacuated and lysis of fibrinopurulent adhesions can be completely operated, while in some cases with a thick pleural peel or a complicated multiloculated pleural space, pleural decortication under thoracoscope can be performed as well [11].

A $28 \mathrm{~F}$ chest tube was inserted at the end of the procedure through the same or the first entry port and chest drain was connected to an underwater drainage bottle. The chest tube can be removed if complete lung re-expansion was confirmed on CXR or fluid drainage was less than $100 \mathrm{~mL} / 24 \mathrm{~h}$. 


\section{Statistical analysis}

The descriptive analysis was expressed as frequency and mean $\pm \mathrm{SD}$. Chi-square test was performed to compare categorical variables and Fisher's exact test was used for small samples. A $p$-value $<0.05$ was considered statistically significant. Bonferroni correction was made for adjusting the effects of multiple comparisons of the variables. Data were analyzed using SPSS 19.0 statistical software (SPSS Inc., Chicago, IL, USA).

\section{Results}

One thousand nine hundred and twenty-six patients were involved in the study with a mean age of $51 \pm 8.4$ years (range 21-86). Characteristics of the patients were shown in Table 1. In patients who had bilateral pleural effusion, MT was performed on the side with larger pleural effusion. The diagnosis of empyema was made by microbiological and biochemical examinations of pleural fluid before MT.

Diagnostic outcomes were represented in Table 2 . Eighty patients were not definitely diagnosed before they discharged from hospital and they were considered as unidentified pleural effusion.

Table 3 revealed complications during and after MT. One 63-year-old male patient diagnosed as empyema died during the creation of pneumothorax before

Table 1 Patients characteristics for medical thoracoscopy $(n=1926)$

\begin{tabular}{ll}
\hline Variable & Value \\
\hline Age (years) & $51( \pm 8.4)$ \\
Mean ( \pm standard deviation) & $21-86$ \\
Range & \\
Gender & $1117(58.0)$ \\
Male & $809(42.0)$ \\
Female & \\
Affected side & $770(40.0)$ \\
Left & $1102(57.2)$ \\
Right & $54(2.8)$ \\
Bilateral & \\
Operation of medical thoracoscopy & $662(34.4)$ \\
Diagnostic procedure & $1264(65.6)$ \\
Therapeutic procedure & $358(28.3)$ \\
Pleurodesis & $517(40.9)$ \\
Adhesiolysis & $125(9.9)$ \\
Pleural decortication of empyema* & $264(20.9)$ \\
Bulla electrocoagulation & \\
Types of instruments & $1586(82.4)$ \\
Rigid medical thoracoscopy & $340(17.6)$ \\
Semi-rigid medical thoracoscopy &
\end{tabular}

*Cases of empyema were diagnosed before medical thoracoscopy;

Values are presented as No. (\%) unless otherwise indicated
Table 2 Diagnostic outcomes of medical thoracoscopy $(n=1926)$

\begin{tabular}{ll}
\hline Diagnosis outcomes & Number (\%) \\
\hline Benign & $986(51.2)$ \\
Pneumothorax & $264(26.8)$ \\
Parapneumonic effusion & $366(37.1)$ \\
Tuberculous pleurisy & $317(32.1)$ \\
Other benign pleural diseases & $39(4.0)$ \\
Malignant & $860(44.6)$ \\
Mesothelioma1 & $48(17.2)$ \\
Pleural metastasis & $712(82.8)$ \\
Unidentified pleural effusion & $80(4.2)$ \\
\hline
\end{tabular}

Values are presented as No. (\%) unless otherwise indicated

thoracoscopy. The patient was given artificial respiration with oxygen; chest compression was performed; coramine and adrenaline were injected intravenously. These efforts were of no avail and the patient died after $30 \mathrm{~min}$. No procedure-related deaths happened in other cases. Hemolytic pleural fluid drainage exceeded $1000 \mathrm{ml} / 10 \mathrm{~h}$ in one patient who was converted to thoracic surgery. In addition, 401 patients $(20.8 \%)$ had a temperature $>$ $37.4^{\circ} \mathrm{C}$ and temperature dropped to normal level with physical cooling or non-steroidal anti-inflammatory drugs (NSAIDS). Of the 137 cases of cutaneous infection in entry site, none resulted in severe empyema or sepsis. Moreover, re-expansion pulmonary edema was reported in two patients with large to moderate amount of pleural effusion. Both patients suffered from refractory cough and dyspnea, and CXR on bedside showed diffuse opacity of the right hemithorax. Dyspnea was improved by high-flow oxygen and IV diuretics, with CXR at $48 \mathrm{~h}$ after MT showing disappearance of the previously observed changes.

Table 3 Complications of medical thoracoscopy $(n=1926)$

\begin{tabular}{ll}
\hline Complications & Number (\%) \\
\hline Major complications & $1(0.1)$ \\
Mortality & $6(0.3)$ \\
Lung laceration & $7(0.4)$ \\
Bleeding & $2(0.1)$ \\
Reexpansion pulmonary edema & $9(0.5)$ \\
Prolonged air leak & $2(0.1)$ \\
Mediastinal emphysema & \\
Minor complications & $62(3.2)$ \\
Subcutaneous emphysema & $749(38.9)$ \\
Pain & $401(20.8)$ \\
Fever & $137(7.1)$ \\
\hline
\end{tabular}

Values are presented as No. (\%) unless otherwise indicated 
Distribution of complications in diagnostic and therapeutic thoracoscopy was illustrated in Fig. 1. Incidence of pain, fever and cutaneous infection in entry site was higher in patients who underwent therapeutic thoracoscopy, according to the data mentioned in Table 4.

Figure 2 described complications of different therapeutic thoracoscopy groups. Comparing with other therapeutic methods, complication of subcutaneous emphysema was more common in adhesiolysis and bulla electrocoagulation groups (Table 5). Higher incidence of prolonged air leak was observed in bulla electrocoagulation group, in comparison with pleurodesis group. Furthermore, pain and fever were the most frequently complications in pleurodesis group and cutaneous infection in entry site was the most frequently reported complication in pleural decortication of empyema group.

\section{Discussion}

Medical thoracoscopy refers to a invasive procedure to be commonly performed as a definitive diagnostic method [14] in undiagnostic exudative pleural effusion, as well as to be widely used in the management of pleural diseases. Many authors claim that MT is a safe procedure with low mortality, in patients who underwent MT for diagnostic thoracoscopy and talc poudrage pleurodesis [18-20]. In our comprehensive research, we found therapeutic interventions of MT increased risks of adverse actions.

One patient, who was diagnosed as empyema, died when artificial pneumothorax was being established. A general examination of the cardiovascular and central nervous systems revealed no abnormalities before MT. While $260 \mathrm{ml}$ atmospheric air was slowly insufflated into pleural cavity, pleural pressure rapidly increased and respiratory-cardiac arrest occurred in the patient. Because of refusal of autopsy, the cause of death is not definitely confirmed. In light of the data collected and the representation of this patient's death, the underlying mechanism is suspected. First of all, it is assumed that air embolism is the cause of death. Chest ultrasonography before MT revealed extensive adhesions in the pleural cavity and rapidly increased intrathoracic pressure could induce tearing of pleural adhesions, which resulted in rupture of veins on pleura. Large amount of air entered the ruptured veins and the bubbles could pass from intercostal veins through the azygos vein system to the superior vena cava, and could kill the patient by producing air locks in the right ventricle. In addition, there is one more point that a range of nerve reflexes stimulated by rapidly increased intrathoracic pressure result in respiratory depression and cardiac arrest. It has been reported in rabbits that increased pressure on the thoracic pleura induces a marked inhibition of phrenic motoneurons and a sympathetic outflow reduction of the circulatory system [21]. We suspect that increased intrathoracic pressure in the patient reduced both the amplitude of phrenic nerve discharge and systolic and diastolic pressure, and resulted in the inhibition of inspiratory activity and cardiac function. To prevent these risks, it is safer to be performed by trained experienced pulmonologists and measurements of lung function are implemented before the procedure. During the creation of pneumothorax, operator should feel and observe negative respiratory fluctuations on the manometer until pleural pressure nears zero, or $800-1000 \mathrm{ml}$ air has been insufflated. Pressure of air insufflation never exceeds $10 \mathrm{cmH} 2 \mathrm{O}$, and inflation should be stopped if the patient is suffering from discomfort or resistance to insufflation is experienced. By this means, air embolism, cardiac disturbances or tearing of pleural adhesions would be avoided. Nevertheless, establishment of artificial pneumothorax in patients with

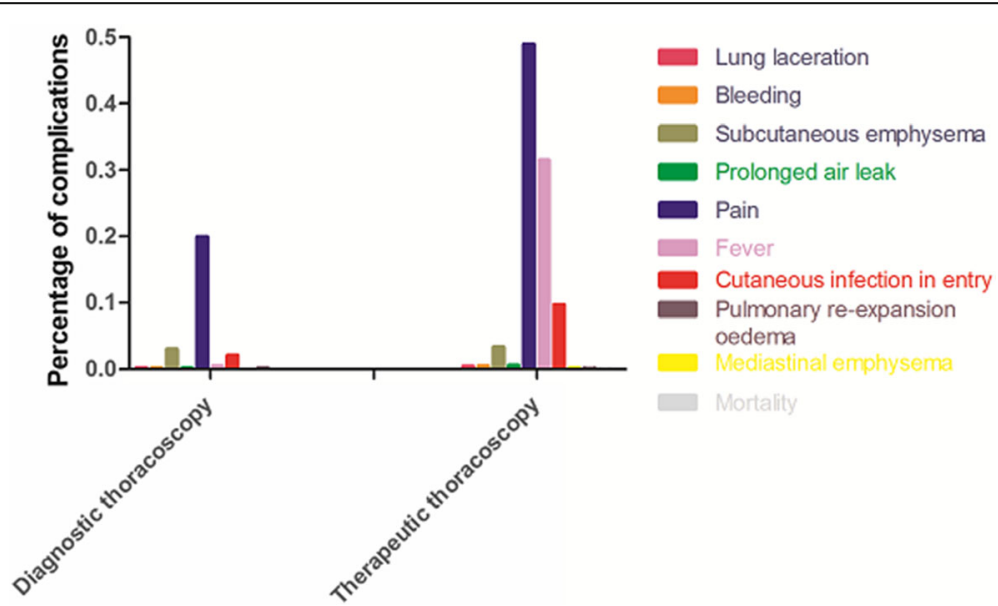

Fig. 1 Distribution of complications in diagnostic and therapeutic thoracoscopy. In diagnostic thoracoscopy group, complications of pain, subcutaneous emphysema and cutaneous infection in entry site were more common; in therapeutic thoracoscopy group, complications of pain, fever and cutaneous infection in entry site were more common 
Table 4 Comparison of complications between diagnostic and therapeutic thoracoscopy $(n=1926)$

\begin{tabular}{|c|c|c|c|}
\hline Complications & $\begin{array}{l}\text { Diagnostic thoracoscopy } \\
(n=662)\end{array}$ & $\begin{array}{l}\text { Therapeutic thoracoscopy } \\
(n=1264)\end{array}$ & $P$-values \\
\hline \multicolumn{4}{|l|}{ Major complications } \\
\hline Mortality & 0 & $1(0.1)$ & 1.0 \\
\hline Lung laceration & $1(0.2)$ & $5(0.4)$ & 0.628 \\
\hline Bleeding & $1(0.2)$ & $6(0.5)$ & 0.47 \\
\hline Pulmonary reexpansion oedema & $1(0.2)$ & $1(0.1)$ & 1.0 \\
\hline Prolonged air leak & $1(0.2)$ & $8(0.6)$ & 0.262 \\
\hline Mediastinal emphysema & 0 & $2(0.2)$ & 0.549 \\
\hline \multicolumn{4}{|l|}{ Minor complications } \\
\hline Subcutaneous emphysema & $20(3.0)$ & $42(3.3)$ & 0.722 \\
\hline Pain & $132(19.9)$ & $617(48.8)$ & $<0.001$ \\
\hline Fever & $3(0.5)$ & $398(31.5)$ & $<0.001$ \\
\hline Cutaneous infection in entry site & $14(2.1)$ & $123(9.7)$ & $<0.001$ \\
\hline
\end{tabular}

Values are presented as No. (\%) unless otherwise indicated

extensive pleural adhesions is a paramount challenge that needs cautious consideration.

Lung laceration is an important complication which happened in 6 patients in whom artificial pneumothorax was not established. All of them occurred during introduction of the trocar and did not exceed $1 \mathrm{~cm}$ in length. During the entire procedure, no air leakage was detected and slight blood streamed from the site of the laceration. All the cases were neglected and no complications happened after MT. If the length of lung laceration exceeds $1 \mathrm{~cm}$ or excessive bleeding and air leakage are discovered, the following treatments can be performed by calling cardiothoracic surgeon and application of absorbable mesh and fibrin glue over the laceration under MT. Establishment of artificial pneumothorax a few hours or 1 day before MT can prevent lung laceration which was confirmed in our study. However, if there is enough pleural fluid, it is safe to introduce a blunt trocar into

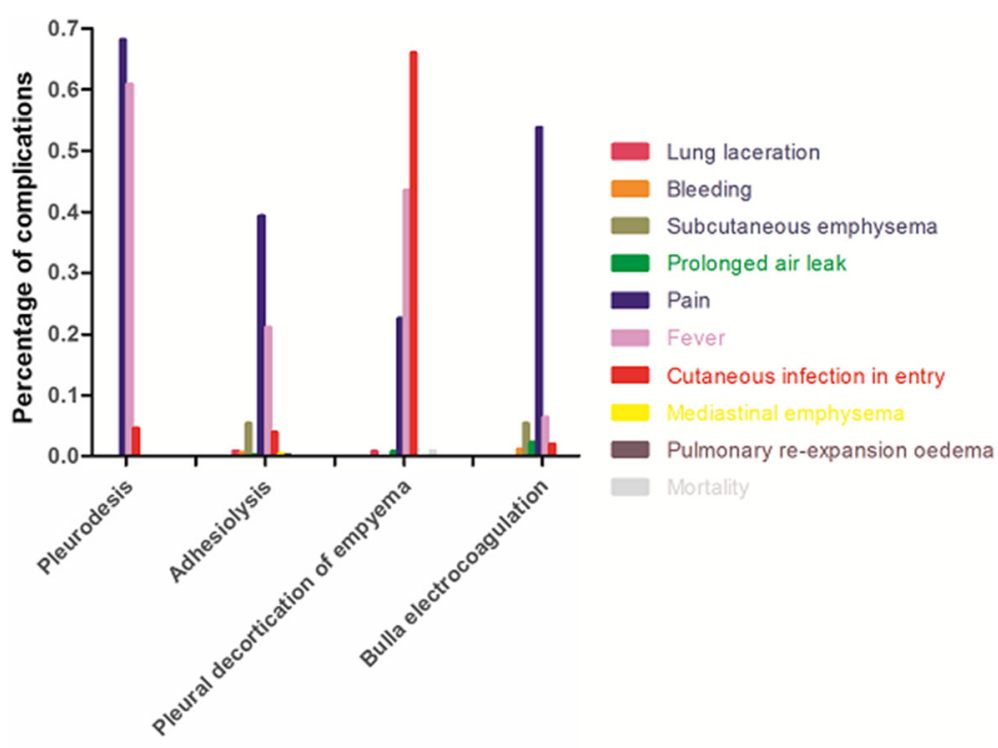

Therapeutic thoracoscopy groups

Fig. 2 Distribution of complications in therapeutic thoracoscopy groups. In pleurodesis group, complications of pain, fever and cutaneous infection in entry site were more common; in adhesiolysis group, complications of pain, fever and subcutaneous emphysema were more common; in pleural decortication of empyema group, complications of cutaneous infection in entry site, fever and pain were more common; in bulla electrocoagulation group, complications of pain, fever and subcutaneous emphysema were more common 
Table 5 Comparison of complications in therapeutic thoracoscopy group ( $n=1264)$

\begin{tabular}{|c|c|c|c|c|c|}
\hline \multirow[t]{2}{*}{ Complications } & \multicolumn{4}{|c|}{ Therapeutic thoracoscopy } & \multirow[t]{2}{*}{$P$-values } \\
\hline & $\begin{array}{l}\text { Pleurodesis } \\
(n=358)\end{array}$ & $\begin{array}{l}\text { Adhesiolysis } \\
(n=517)\end{array}$ & $\begin{array}{l}\text { Pleural decortication of empyema } \\
(n=125)\end{array}$ & $\begin{array}{l}\text { Bulla electrocoagulation } \\
(n=264)\end{array}$ & \\
\hline \multicolumn{6}{|l|}{ Major complications } \\
\hline Mortality & 0 & 0 & $1(0.8)$ & 0 & 0.099 \\
\hline Lung laceration & 0 & $4(0.8)$ & $1(0.8)$ & 0 & 0.158 \\
\hline Bleeding & 0 & $3(0.6)$ & 0 & $3(1.1)$ & 0.185 \\
\hline Pulmonary re-expansion oedema & 0 & $1(0.2)$ & 0 & 0 & 1.0 \\
\hline Prolonged air leak & 0 & $1(0.2)$ & $1(0.8)$ & $6(2.3)$ & $0.002 *$ \\
\hline Mediastinal Emphysema02 & 0 & $2(0.4)$ & 0 & 0 & 0.597 \\
\hline \multicolumn{6}{|l|}{ Minor complications } \\
\hline Subcutaneous emphysema & 0 & $28(5.4)$ & 0 & $14(5.3)$ & $<0.001^{*}$ \\
\hline Pain & $244(68.2)$ & $203(39.3)$ & $28(22.4)$ & $142(53.8)$ & $<0.001^{*}$ \\
\hline Fever & $218(60.9)$ & $109(21.1)$ & $54(43.2)$ & $17(6.4)$ & $<0.001^{*}$ \\
\hline Cutaneous infection in entry site & $16(4.5)$ & $20(3.9)$ & $82(65.6)$ & $5(1.9)$ & $<0.001^{*}$ \\
\hline
\end{tabular}

Values are presented as No. (\%) unless otherwise indicated

* Significance after adjustment with Bonferroni correction $(P<0.0083)$

the thoracic cavity without prior creation of pneumothorax [22]. Chest ultrasonography and CT can be very helpful to identify pleural adhesions in chest wall and locate an appropriate entry site for thoracoscopy [9]. In our study, both chest ultrasonography and CT scan were done in the 6 cases with lung lacerations and a blunt trocar was used, the main causes were probably to be vigorous maneuver during introducing the trocar and absence of artificial pneumothorax. Furthermore, in this study, four of the six patients (66.7\%) were in adhesiolysis group. It indicates that extensive adhesions in pleural cavity increase the risk of lung laceration when introducing a trocar, and location with chest US or CT before MT is not a guarantee of security. What degree of adhesions is insufficient for MT? What is the selection for patients with extensive adhesions, VATS or other pleural procedures? These crucial issues should be resolved in the future.

Re-expansion pulmonary edema (RPE) is a rare and potentially lethal complication and it occurred in two patients in our study. With regard to the first patient, a volume of $2000 \mathrm{ml}$ pleural effusion was aspirated from pleural cavity during MT, and collapsed right lung reexpanded rapidly. Although exact pathogenesis of RPE is still not fully understood, some pathogenic factors for RPE have been reported, such as rapid reexpansion, airway obstruction, longer duration of lung collapse, decreased surfactant activity and increased pulmonary vascular permeability due to injuries to the pulmonary micro-vessels [23]. Regarding to the second patient with moderate amount of pleural effusion, the causes of RPE were considered as longer duration of lung collapse and rapid reexpansion after adhesiolysis. Re-expansion pulmonary edema is usually seen in ipsilateral lung but contrlateral or bilateral cases also have been reported [23]. Treatments of RPE include high-flow oxygen and IV diuretics. Other intensive therapies, such as circulation support, steroid administration, and mechanical ventilation, are required in severe cases of RPE $[24,25]$. Repeated aspirations of air or pleural fluid to facilitate lung re-expansion before MT and use of antibechic after the procedure can prevent occurrence of RPE.

With respect to developing bleeding and chest pain, massive hemorrhage usually occurred when mechanical lysis of adhesions was performed in patients with pneumothorax. Under MT, abundant and thick blood vessels were observed in adhesion zones between the visceral and parietal pleura. We are conducting experiments to study structure and origination of the blood vessels. In patients with pneumothorax, adhesiolysis with electrothermal forceps is more favourable, instead of mechanical lysis. Pain was the most common complication in this study and can be well tolerated by most of patients. Severe pain usually happened when pleurodesis was conducted during MT and sometimes transient attack of hypotension happened due to violent pain. The procedure should be stopped and blood pressure can be elevated to normal range without medicine treatment. Blood pressure, heart rate and oxygen saturation should be monitored during pleurodesis, especially when the procedure is performed in patients with hypovolemia or cardiac insufficiency.

Prolonged air leak was more common in bulla electrocoagulation group. It is probably related to destruction of emphysematous bullae, and it is advised that bulla electrocoagulation combined with pleurodesis would reduce incidence of prolonged air leak. 
In addition, the current study found that pleurodesis group had the highest incidence of fever, which was probably in relation to a systemic inflammation inducing by intrapleural insufflation of sclerosing agents. Steroids and NSAIDS could reduce the quality of pleurodesis [26-29], therefore physical cooling is recommended. With respect to developing cutaneous infection in entry site, it can be modified by improving patient management after the procedure, including dressing change regularly and the use of intravenous antibiotic.

One strengthen of the current research is that a large size of study population is investigated, in which 1926 patients are included. On the other hand, we have to admit that there are some limitations in our research. This is a retrospective study of practice and outcome, and perspective design is under consideration. Follow-up does not continue after patients discharge from hospital, therefore late complications are not represented in the study.

\section{Conclusions}

In conclusion, when medical thoracoscopy is performed by experienced physicians, MT under local anesthesia is generally a safe and effective method for the diagnostic and therapeutic procedures. Mastering medical thoracoscopy well, improving patient management after the procedure and attempts to reduce the occurrence of post-procedural complications should be encouraged.

\section{Abbreviations \\ COPD: Chronic obstructive pulmonary disease; CT: Computed tomography; CXR: Chest X-ray; ECG: Electrocardiogram; IV: Intravenous; MT: Medical thoracoscopy; NSAIDS: Non-steroidal anti-inflammatory drugs; RPE: Re- expansion pulmonary edema; US: Ultrasonography; VATS: Video-assisted thoracoscopic surgery}

\section{Acknowledgements}

We thank the staff at the Department of Respiratory Medicine of Shandong Provincial Hospital affiliated to Shandong University for their assistance in performing clinical operations and collecting clinical data. There was nonfinancial support in our article.

\section{Authors' contributions}

YYW, CCZ, XSL, QHL, and DJL designed this study. CCZ, XSL, ZHY, DZL and NW were involved in the acquisition of the data. CCZ, XSL, YYW, LZ, XLL and DJL analysed the data. CCZ, YYW, XSLZHY, QHL and LZ wrote the manuscript. All authors read and approved the final manuscript.

\section{Funding}

Not applicable.

\section{Availability of data and materials}

The datasets used during the current study are available from the corresponding author on reasonable request. Due to the privacy policy of patients, A truncated dataset after eliminating all potentially identifiable features may be provided on an individual request basis.

\section{Ethics approval and consent to participate}

The Ethics Committee of Shandong Provincial Hospital affiliated to Shandong University approved the study which was conducted according to the principle expressed in the Declaration of Helsinki and the number was NO.2017-225. All patients have provided written informed consents.
Consent for publication

Not applicable.

\section{Competing interests}

The authors declare that they have no competing interests.

Received: 17 January 2018 Accepted: 1 July 2019

Published online: 10 July 2019

\section{References}

1. Rodriguez-Panadero F, Antony V. Pleurodesis. State of the art. Eur Respir J. 1997;10:1648-54.

2. Gordon S. Clinical reports of rare cases, occurring in the Whitworth and Hardwicke hospitals. Dublin Q J Med Sci. 1866;41:83-90.

3. McLean AN, Bicknell SR, McAlpine LG, Peacock AJ. Investigation of pleural effusion: an evaluation of the new Olympus LTF semiflexible thoracofiberscope and comparison with Abram's needle biopsy. Chest. 1998;114:150-3.

4. Loddenkemper R. Thoracoscopy--state of the art. Eur Respir J. 1998;11:213-21.

5. Mathur PN, Loddenkemper R. Medical thoracoscopy. Role in pleural and lung diseases. Clin Chest Med. 1995;16:487-96.

6. Boutin C, Viallat JR, Cargnino P, Farisse P. Thoracoscopy in malignant pleural effusions. Am Rev Respir Dis. 1981;124:588-92.

7. Menzies R, Charbonneau M. Thoracoscopy for the diagnosis of pleural disease. Ann Intern Med. 1991:114:271-6.

8. Loddenkemper R, Boutin C. Thoracoscopy: present diagnostic and therapeutic indications. Eur Respir J. 1993;6:1544-55.

9. Rozman A, Camlek L, Marc-Malovrh M, Triller N, Kern I. Rigid versus semirigid thoracoscopy for the diagnosis of pleural disease: a randomized pilot study. Respirology (Carlton, Vic.). 2013;18:704-10.

10. Tschopp JM, Rami-Porta R, Noppen M, Astoul P. Management of spontaneous pneumothorax: state of the art. Eur Respir J. 2006;28:637-50.

11. Tassi GF, Davies RJ, Noppen M. Advanced techniques in medical thoracoscopy. Eur Respir J. 2006;28:1051-9.

12. Colt HG. Thoracoscopy: A prospective study of safety and outcome. Chest. 1995;108:324-9

13. Lee P, Hsu A, Lo C, Colt HG. Prospective evaluation of flex-rigid pleuroscopy for indeterminate pleural effusion: accuracy, safety and outcome. Respirology (Carlton, Vic). 2007;12:881-6.

14. Rahman NM, Ali NJ, Brown G, et al. Local anaesthetic thoracoscopy: British Thoracic Society pleural disease guideline 2010. Thorax. 2010;65(Suppl 2):ii54-60.

15. Lee P, Colt HG. Rigid and semirigid pleuroscopy: the future is bright. Respirology (Carlton, Vic.). 2005;10:418-25.

16. Alraiyes AH, Dhillon S, Harris K, Kaphle U, Kheir F. Medical Thoracoscopy: Technique and application. Pleura. 2016:3:1-11.

17. Alpay Orki RD, Ciftci H, Coskun T, Asim Kutlu C, Arman B. Videothoracoscopic approach to recurrence primary spontaneous pneumothorax: using of electrocoagulation in small bulla/blebs. Indian J Surg. 2009:71:19-22.

18. Brims FJ, Arif M, Chauhan AJ. Outcomes and complications following medical thoracoscopy. Clin Respir J. 2012;6:144-9.

19. Rodríguez-Panadero F. Medical thoracoscopy. Respiration. 2008;76:363-72.

20. Shimaa NMAHS, Ali MH, et al. Safety and complications of Medical Thoracoscopy. Advances in Medicine. 2016;10:1-6.

21. Jammes $Y$, Delpierre $S$. Respiratory and circulatory effects of parietal pleural afferent stimulation in rabbits. J Appl Physiol. 2006;100:1539-46.

22. Faurschou P, Viskum K. Artificial pneumothorax by the Veress cannula: efficacy and safety. Respir Med. 1997:91:402-5.

23. Kim JJKY, Choi SY, Jeong SC, Moon SW. Contralateral reexpansion pulmonary edema with ipsilateral collapsed lung after pleural effusion drainage: a case report. J Cardiothorac Surg. 2015;10:68.

24. Kim YK, Kim H, Lee CC, Choi HJ, Lee KH, Hwang SO, Oh JH, Lee YH, Singer AJ. New classification and clinical characteristics of reexpansion pulmonary edema after treatment of spontaneous pneumothorax. Am J Emerg Med. 2009:961-967:590-602.

25. Taira N, Kawabata T, Ichi T, Yohena T, Kawasaki H, Ishikawa K. An analysis of and new risk factors for reexpansion pulmonary edema following spontaneous pneumothorax. J Thorac Dis. 2014;6:1187-92.

26. Teixeira LR, Vargas FS, Acencio MM, Paz PF, Antonangelo L, Vaz MA, Marchi E. Influence of antiinflammatory drugs (methylprednisolone and diclofenac 
sodium) on experimental pleurodesis induced by silver nitrate or talc. Chest. 2005;128:4041-5.

27. Hunt I, Teh E, Southon R, Treasure T. Using non-steroidal anti-inflammatory drugs (NSAIDs) following pleurodesis. Interact Cardiovasc Thorac Surg. 2007;6:102-4.

28. Gilleece MH, Evans CC, Bucknall RC. Steroid resistant pleural effusion in systemic lupus erythematosus treated with tetracycline pleurodesis. Ann Rheum Dis. 1988;47:1031-2

29. Rahman NM, Pepperell J, Rehal S, Saba T, Tang A, Ali N, et al. Effect of opioids vs nsaids and larger vs smaller chest tube size on pain control and pleurodesis efficacy among patients with malignant pleural effusion: the time1 randomized clinical trial. Jama. 2015;314:2641-53.

\section{Publisher's Note}

Springer Nature remains neutral with regard to jurisdictional claims in published maps and institutional affiliations.

Ready to submit your research? Choose BMC and benefit from:

- fast, convenient online submission

- thorough peer review by experienced researchers in your field

- rapid publication on acceptance

- support for research data, including large and complex data types

- gold Open Access which fosters wider collaboration and increased citations

- maximum visibility for your research: over $100 \mathrm{M}$ website views per year

At BMC, research is always in progress.

Learn more biomedcentral.com/submissions 